\title{
Proactive management of innovations in information technology (IT) enabled services
}

\author{
Ada Hui-Chuan Chen ${ }^{1 \star}$, Rua-Huan Tsaih ${ }^{2}$, Hsin-Lu Chang ${ }^{2}$ and Shrane-Koung Chou ${ }^{2}$ \\ ${ }^{1}$ Department of Information Management, Ching Yun University, Zhongli, Taoyuan, Taiwan, Republic of China. \\ ${ }^{2}$ Department of Management Information Systems National, Chengchi University, Taipei City, Taiwan,
} Republic of China.

Accepted 22 August, 2012

\begin{abstract}
Since the early 1960s, many innovations on information technology-enabled services (IISs) that fulfill underserved customers and create competitive advantages for firms have been examined on the realization of their values. However, even with much theoretical promise, most IIS experiments have failed. Extant arguments attribute this to initiatives, business models, institutional challenges, professions, sector characteristics, market segments or geographic locations. However, the issue on how to proactively manage the development, deployment and diffusion of new IT-enabled services still remains unclear. This study provides a comprehensive picture of (potential) challenges of IISs, explains how values and expenses are transformed within IIS projects. Moreover, we can conclude that elaborate IT-enabled services enable service-for-service exchanges and produce innovations and challenges. Nevertheless, successful IT-enabled services need not only to eliminate retardants from different levelssocietal, industrial, organizational, group and individual, but also to maximize values while controlling expenses during the process of IISs.
\end{abstract}

Key words: Service, service innovation, information technology-enabled service, proactive management, value.

\section{INTRODUCTION}

Innovations in information technology-enabled services

In the academic literature, service has been elaborated on by scholars from different perspectives and as the role of services becomes increasingly significant in today's economy, its fundamental definition has undergone evolutionary changes. Early studies describe the essence of services as yielding satisfaction, perishability, simultaneity and intangibility (Regan, 1963; Sasser et al., 1978). Subsequent researchers assert that services are deeds, processes and performances for providing valueadded solutions to customer needs (Gronroos, 1990; Zeithaml and Bitner, 1996). The latest researchers underscore the fact that services are capabilities or competencies that enterprises provide for customers and the process of co-production, participation and value creation is emphasized (Lovelock and Wirtz, 2010; Sheth et al., 2006; Vargo and Lusch, 2004). In addition, "the appetite for services, especially innovative ones, is insatiable" (Fitzsimmons and Fitzsimmons, 2008). On the other hand, information technologies (IT) (including communication and networking) and thus information applications (IA) in all sectors have evolved dramatically in the digital age. Along with globalization, service innovation and IT/IA evolution bring about (incremental or radical) innovations in IT-enabled services that fulfill underserved customer needs and create competitive advantages for firms.

The concept of IT-enabled services (ITeS) has been defined by different researchers. Some of them are: 
1. An ITeS is the combination of knowledge regarding certain specific areas and IT, or the generation of new application ideas based on IT itself, to create innovative technology-based services, new business models and value-added services (MOEA, 2006).

2. An ITeS is the capabilities, structures and processes with which digitized services are conceived and architected, how these services are offered and orchestrated, and how interactions for innovation and production of services are managed" (Rai and Sambamurthy, 2006).

3. An ITeS is information-abundant and fulfilled through IAs that function intra-organizationally and interorganizationally; it is expected to have more automation, self-service, and intelligence within the service process (Apte and Goh, 2004).

In general, ITeS emphasize an IT empowerment approach that allows the customer to assume a new and perhaps more independent, role as an active participant in the self-service process. ITeS also can allow employees to interact with each other across functional boundaries and even with those in other firms, all in "real time" and without the need to be physically present.

Since the early 1960s, many innovations in ITeS (IISs), such as automatic teller machines, interactive television services, on-line shopping services and IT-enabled selfservices on securities brokerages, have been tested to assess the materialization of their values. Theoretically, IIS value consists of ValueC, the value delivered to the customer and ValueCA, the competitive advantage delivered by IISs to the firm. The value of a service from the customer's perspective can be captured in Equation (1) (Heskett et al., 1997). Thus, ValueC is related to the degree of new services that address the customer needs of a reference group, the process quality of the ways in which new services are delivered, the alignment between perceptions and expectations of the reference group regarding new services, and the costs of acquiring new services. Hereafter, the reference group refers to the customers of the perceived market segment.

Valuec $=\frac{\text { Resultsproduced for the customer }+ \text { Process quality }}{\text { Price to the custmer }+ \text { Costs of acquiring the service }}$

The theoretical ValueCA is based on three elements. The first is product differentiation resulting from new combinations of information, products and services and the reconfiguration and integration of resources, capabilities, roles and relationships among partners and customers. The second is the investment of resources that are complementary to ITeS (for example IT assets, new organizational forms or business processes, ITrelated intangibles) (Teece, 1986). Firms that make such investments can experience positive changes in market value because such investments provide competitive advantages that are difficult for competitors to duplicate.
The third is new business opportunities brought by commercialized ITeS such as new information chains that support the new services (Fitzsimmons and Fitzsimmons, 2008; Karmarkar and Apte, 2007).

Even with much theoretical promise (in ValueC and ValueCA), however, most IIS experiments have failed and faded away. It seems that there is no guarantee for the prosperity of IISs. Perhaps, this might be explained by the fact that IISs are normally initiated from either marketplace studies or IT-laboratory studies. Marketplace studies provide some ideas about underserved customers' needs but offer little information about immediate IT/IA solutions. On the other hand, ITlaboratory studies lead to some creative IT/IA but offer little information on solutions via business models, collaborations, and work practices in service provisions. Only IISs initiated from marketplace studies with immediate IT/IA solutions or from IT-laboratory studies with the right management/market solutions lead to prosperous businesses, if the timing is right. Such predicaments may be avoided via proactively managing the process of developing, deploying and diffusing a new ITeS, which we call three-Ds of IIS.

Unfortunately, we find that there is no comprehensive study in the literature that explores the proactive management of three-Ds of IIS. Thus, in this study, we aim to help managers foresee possible challenges associated with three-Ds of IIS (that is, potential retardants that discount the value of new service or, in extreme cases, lead to termination) and identify key issues regarding the proactive management of three-Ds of IIS.

Specifically, there are three objectives of the study. First, we conducted a literature review in services management and operations management to obtain an ontological description of three-Ds of IIS. Second, the following two case studies were conducted to derive a general framework for proactively managing three-Ds of IIS: (1) A library case study of Time Warner Cable; and (2) a field case study of Taiwan Television Enterprise, Ltd. (TTV), which is the first terrestrial TV broadcasting company in Taiwan. Both case companies deploy IIS in the TV platform (that is, interactive television service innovations) and encounter challenges involved with three-Ds of IIS. Therefore, evidence collected in both cases is useful to produce a comprehensive picture delineating three-Ds of IIS. Third, and most important, to identify the key issues in the proactive management of three-Ds of IIS, we explore the reasoning behind decisions taken to cope with the identified challenges in the aforementioned case studies.

\section{THE ONTOLOGICAL DESCRIPTION OF THREE-DS OF IIS}

Review works in the literature (Bruhn and Georgi, 2006; Fitzsimmons and Fitzsimmons, 2008; Karmarkar and 


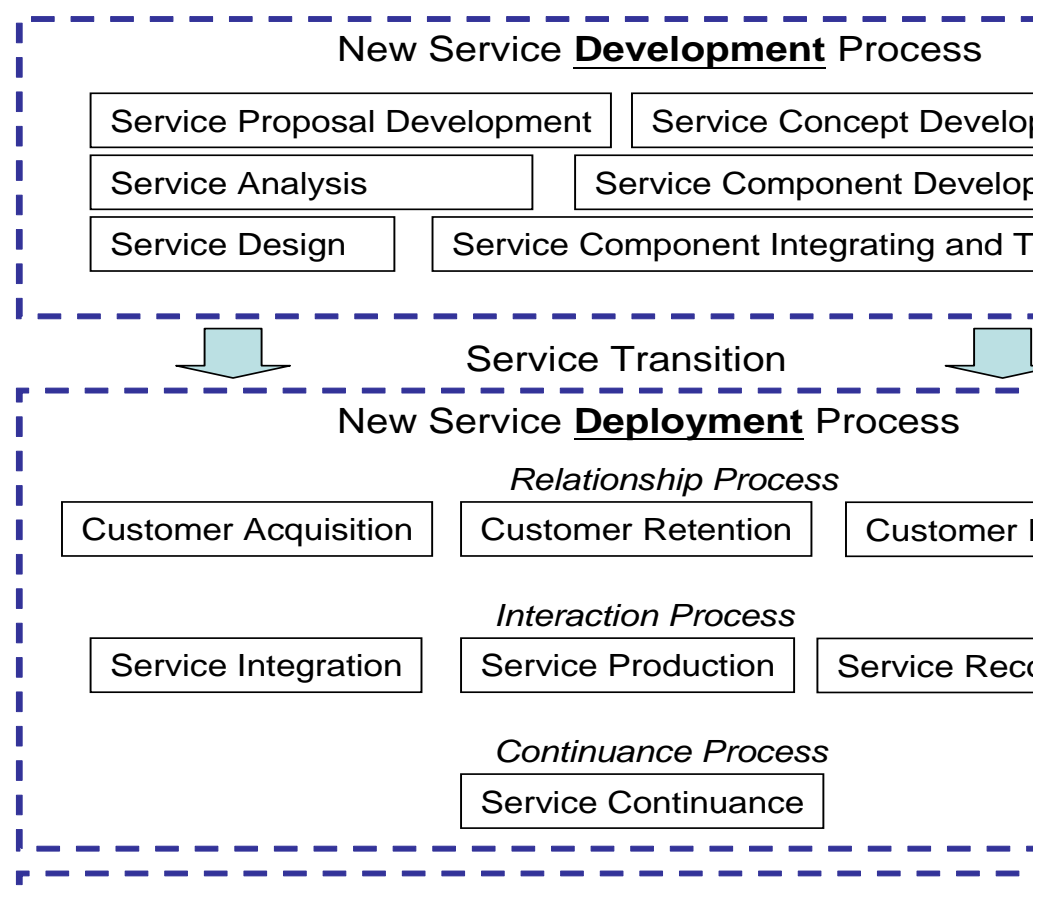

Figure 1. A description of activities in three-Ds of IIS.

Apte, 2007; Meyer and Fahnrich, 2009) conclude activities involved in three-Ds of IIS (Figure 1).

In the development stage, triggered by certain (external or internal) studies, a firm may set up several IIS proposals for ideal service innovation in the beginning instead of random trial and error. For each proposal, a service concept should be developed to provide a more detailed description of a new service system. The service concept should include a well-defined business model on a perceived market segment of customers. Then, the service analysis will determine the potential of the new service as part of a profitable business venture and the top management level will approve the project accordingly. For an authorized IIS proposal, a service design should be assessed to figure out more detailed requirements (for example, service standards, specifications of IT/IA, and standard operation procedures) of service components that include IT assets, people, knowledge and know-how involved in the operations of front/middle/back offices, in which the front office is the portion of service system where customer contact occurs and the back office (and the middle office) is the portion hidden from the customers.

Based on the perceived service design, each service component should be developed and tested. Within service component integration and testing, the firm should set up a pilot service system and infuse acquired IT assets into the business functions to test the synergies of the integrated service components. Generally, the key issues are intangible components of IISs, such as employee acceptance (that is, those involving cultures, incentives and organizational issues), processes of management/reengineering (that is, those enforcing cooperation between inter-functions or setting up new departments), and the acceptance of the reference group.

Once the new service is ready to commence, there is a service transition that transits the pilot service system into a real service system with a full-scale (both internal and external) food chain of service. As mentioned in Bruhn and Georgi (2006), and Fitzsimmons and Fitzsimmons (2008), the new service implementation involves at least (1) the interaction process, in which an interaction describes the throughput of a customer via an individual service process; (2) the relationship process, where the relationship concerns the customer's throughput through a relationship with the service provider; and (3) the continuance process. The interaction process is comprised of service integration, service production, and service recovery.

The relationship process also includes three activities, namely, customer acquisition, customer retention, and customer recovery. The continuance process focuses on the service continuance activities that enhance the customer retention rate by improving the quality of current services, adding extra functions to the current services, or introducing new services. Finally, the activities in the diffusion group specify how the service initiative is commercialized by translating competencies into business performance. 


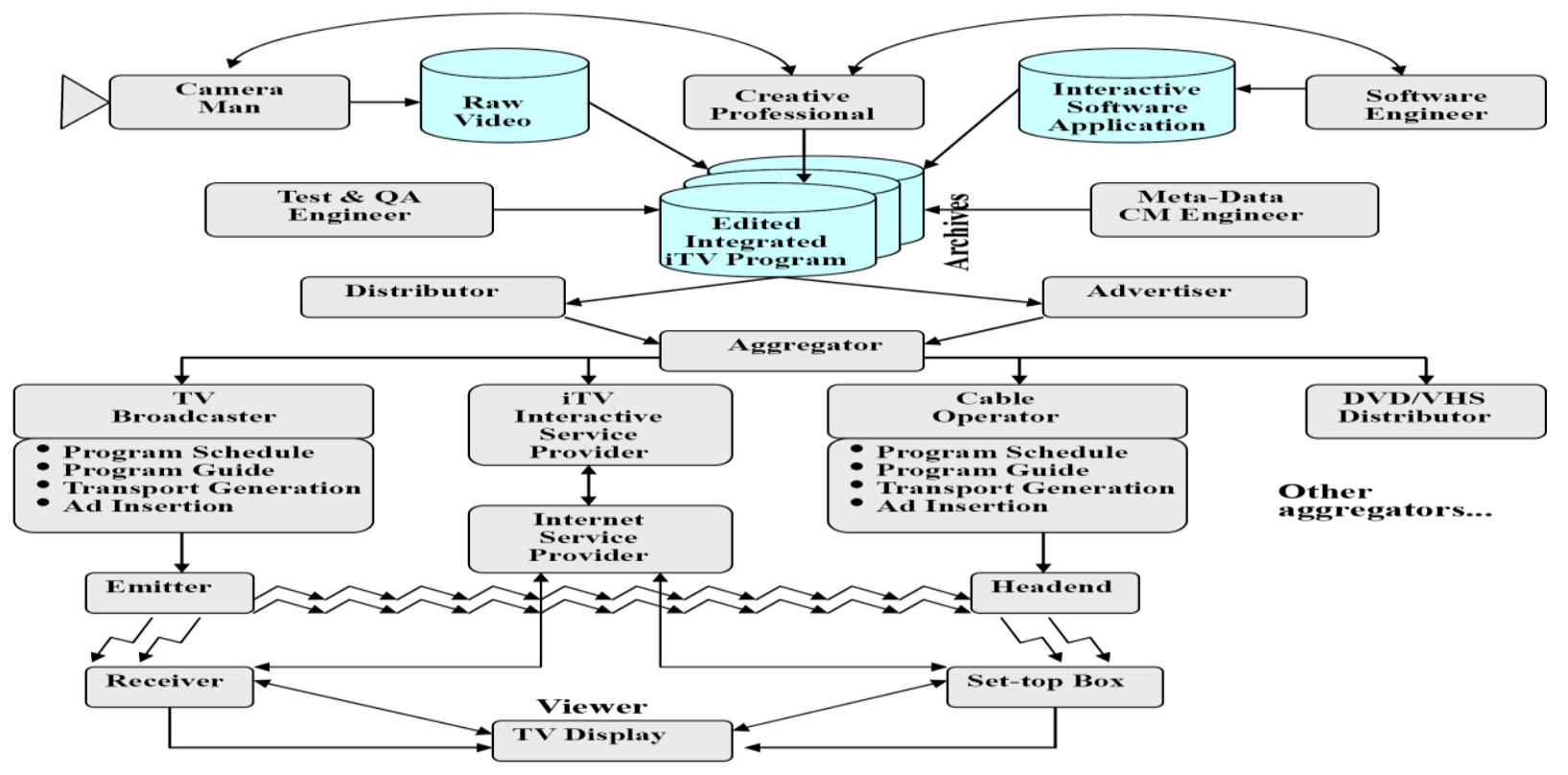

Figure 2. The content production food chain of iTV service adapted from Schwalb (2004).

\section{CASE STUDIES OF INNOVATION OF INTERACTIVE TV SERVICE}

To help identify challenges from the case studies involving Time Warner and Taiwan Television Enterprise (TTV), the complexities of the content production food chain of interactive TV (iTV) services are shown in Figures 2. The food chain starts from the producing/authoring stage, which involves video making and software application. In the integration stage, backand-forth interactions between creative professionals, camera men and software engineers are necessary but time consuming. In addition, the quality assurance and testing engineers and the configuration management professionals are roles that ensure adequate content and well-organized archives. Then, the iTV programs are passed to distributors or advertisers in the releasing stage. The last stage of the iTV food chain is the viewing stage, which involves the interactivity of either a local type without outside communication or a remote type with a bi-directional interactive channel. Note that one of distinctive characteristics between video-on-demand (VOD) services on the Internet and iTV services is that the latter is more constrained by media commercials. Since media commercials are targeted to meet the entertainment needs of viewers and thus improve the size of the viewing audience, iTV services are often based on demographic, lifestyle and psychographic analyses of their subscribed clients who are in specific geographic locations (Tsaih et al., 2005). In sum, the content production food chain of iTV services requires lots of collaboration at the intra-organizational and/or inter-organizational levels.

\section{Time warner cable in the United State}

We conducted a library case study that analyzes relevant archival records and documentary information of time warner cable found in EBSCO Business Complete, corporate website, and other references from the Internet. In 1977, some iTV services were launched by Warner Cable via its QUBE system in Columbia, Ohio. To assess its value in providing interactive ratings, home security, TV shopping, remote education, among other areas, a series of pilot tests was conducted in large cities (that is, Houston, Milwaukee, Dallas, Cincinnati, St. Louis and Pittsburgh). Although the pilot tests were successful, the high cost of building such closed-circuit television systems presented challenges requiring lower-cost technologies, a profitable business model, and high customer acceptance. By 1982, Warner Cable was running a $\$ 99$ million loss, and by 1983 , the total loss amounted to $\$ 418$ million. Moreover, after Warner bought out American Express in the early 1980s, the organization was realigned in such a way that the original development team left and the earlier alignment between the technology and business sides was phased out. At that time, Warner Amex had two million subscribers, roughly $1 / 10$ of the entire cable subscriber market (Warner Communication Annual Report, 1983). The question of whether iTV services for such a broad customer base are profitable became a pressing issue.

Due to such unsolved challenges, Warner Amex 
decided not to move forward with iTV programs and sold the relevant IT assets to Viacom (Britannica, 2010).

Time Warner did not focus on iTV services again until a group of its engineers tried an experimental design of a two-way cable TV system in 1987 (Farhi and Corcoran, 1994). Riding the wave of fiber optic networks and digital computer technology, Time Warner Cable decided to invest $\$ 5$ billion over five years for the full service network (FSN) in 1994, an interactive video service offering shopping, games, sports, news, and movies. The partial rollout of the system began in December 1994, eight months later than the expected rollout date due to technological hurdles. Furthermore, in 1995 and 1996, the development team conducted several different applications on different platforms to explore how advertisers could use FSN. However, an experiment showed that users only ordered, on average, two movies a month through the network. By retrospectively, analyzing the historical evidence, some technical glitches were identified at that time. Specifically, system complexities remained a main issue, such as real-time compression, updated assets and memory limitation of set-top-boxes (Blank, 1995). One technical manager described the situation: "because of equipment shortages, getting a news tape edited, digitized, compressed and loaded on the system takes nearly a week, by which time it is no longer news" (Elmer-Dewitt and Woodbury, 1994)

Eventually, the high cost of the set-top-boxes, low usage rates, and low revenues resulted in its failure. Time Warner announced the phase-out of FSN in mid1997 with only a quick mention that FSN played a key role in "providing the technical and marketing data" (Wikipedia, 2011). Even Time Magazine displayed disapproval, by denoting FSN as "the most expensive pizza delivery system ever invented" (Baumohl and Greenwald, 1997).

The lessons from the FSN case guided Time Warner and other major cable companies in their fundamental technical development of iTV and led to a subsequent rollout of digital cable, high-definition VOD, and ITV guides. Time Warner was the first to move to digital in 1998, followed by Comcast in 2000. Time Warner and Comcast licensed the Gemstar-TV guide in 2001. Time Warner and other major cable companies also formed a joint venture in 2004 to implement the OpenCable application Platform (OCAP), a middleware platform that enables 'plug-and-play' via set-top-free digital TV. These experiences show that it is critical to (1) form a reliable business-IT integration plan that helps to decide the breadth and depth of the invested IT assets and (2) find a way of systematically scrutinizing the iTV projects.

\section{Taiwan television enterprise (TTV) in Taiwan}

A field case study with an in-depth interview with the chairman and CEO of TTV was conducted. From the interview, the chairman and CEO of TTV recognized his proposed ITV services as a commercial exploitation without cutting-edge technology acquisition, since TTV's technology department had attempted to modify and incorporate the interactive technology currently available on the Internet to develop the required IT/IA through government funding. The technology department had designed an on-the-job training program to upgrade employees' technical and managerial skills in the use of relevant IT/IA. Whereas, observing no specific challenges regarding the acquisition of enabling IT assets, the chairman stated that "the most valuable resource in our company is the employees, especially in the technology department." However, these IT assets have not successfully translated into a commercialized service, due to some foreseeable challenges in the managerial, organizational, inter-organizational, and social/environmental dimensions shown in Table 1.

For instance, the chairman found that it was hard to convince the board that the proposed ITV service will generate a feasible economic return in a short period of time and there was lack of necessary infrastructure, such as appropriate training programs and marketing of the innovation across the company to set up a supportive culture that values iTV services. Such a supportive culture would help to ease changes posed by the internal content production. Other necessary organizational investments should include the establishment of a strong team for producing the iTV content/services, the establishment of an efficient process for to provide iTV services and the creation of a strong information systems team and facilities for viewership analysis.

In addition to the previous internal issues, some external issues also concerned the chairman regarding the diffusion of iTV services. For instance, the chairman stressed that the uncertainty of viewer acceptance hindered the commercial exploitation of an iTV service. According to the chairman, persuading viewers to accept iTV content and services is a social challenge that cannot be resolved by a single firm alone, but rather by society at large, as well as governments, other firms, and other key market actors. For instance, the chairman was concerned about government regulation of pricing structures for iTV services, as viewers may be resistant to any changes away from free terrestrial TV programs and low fees for cable TV programs. In addition, publicinterest associations and the government strictly regulate TV programming. Thus, persuading the government to enact regulations and laws that encourage iTV service innovation is a complex social challenge.

The chairman was further concerned about iTV services with regard to the establishment of strategic networks among computer, television, and telecommunication industries. As shown in Figures 2, iTV programming requires significant service enhancements by the participating firms in strategic networks and thus 
Table 1. Challenges to TTV's commercial exploitation of an iTV service.

\begin{tabular}{ll}
\hline Dimension & Challenge \\
\hline Social/environmental & $\begin{array}{l}\text { Viewer acceptance of the iTV service } \\
\text { Laws and regulations that create a fair, stable market environment } \\
\text { iTV infrastructure } \\
\text { Technology standards for the iTV service } \\
\text { Educational programs for raising iTV literacy }\end{array}$ \\
Inter-organizational & $\begin{array}{l}\text { Collaborative work environments for the iTV service } \\
\text { Good governance }\end{array}$ \\
& $\begin{array}{l}\text { Board's approval } \\
\text { Strong senior management support for the iTV service } \\
\text { Incentives for the iTV service }\end{array}$ \\
Managerial & $\begin{array}{l}\text { Teamwork environments for the iTV service } \\
\text { Training programs to enhance the skills for the iTV service }\end{array}$ \\
& $\begin{array}{l}\text { Supportive culture that values the iTV service } \\
\text { Strong development teams for innovative applying the iTV } \\
\text { Efficient process for providing the iTV service } \\
\text { Strong teams and good information systems for the data analysis of viewership opinions }\end{array}$ \\
\hline
\end{tabular}
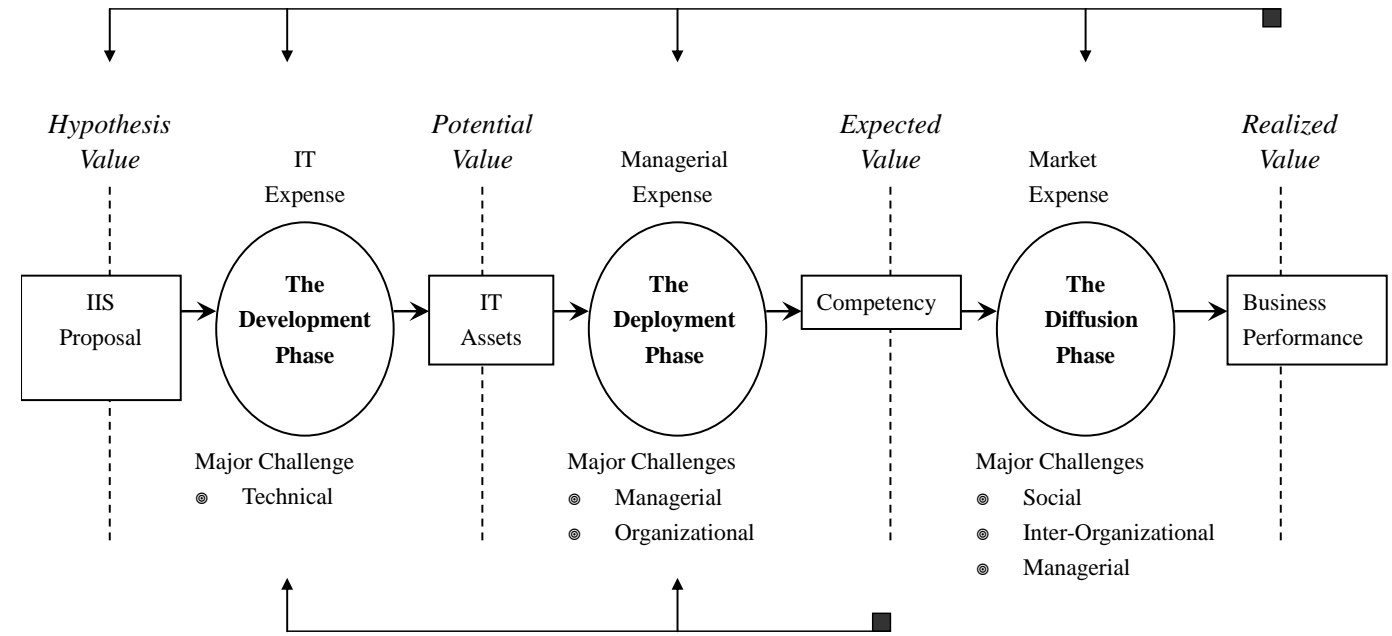

Figure 3. The proposed framework for proactively managing three-Ds of IIS.

requires additional commitment and expertise from other firms. Consequently, the chairman indicated that in order to make iTV services a reality, all of the involved firms need to be willing to share their specialized knowledge and expertise. In addition, governance for iTV service partners must be established through legal contracts or through self-enforcing agreements. Self-enforcing agreements require "formal" safeguards such as financial and investment guarantees, while "informal" safeguards involve goodwill, trust, and reputation. However, such inter-organizational collaboration is a significant challenge for any broadcasting company.

\section{THE PROPOSED FRAMEWORK FOR THREE-DS OF IIS MANAGEMENT}

The identified challenges in the previous cases are further related to activity categories identified in Figure 1. A process-oriented approach (Kohli and Sherer, 2002; Soh and Markus, 1995) is adopted to conceptualize the conversion from the initiation of an IIS to the business performance, as a process of transformation with three phases shown in Figure 3. The main outcomes of the activities (and also the challenges) in the phases of IIS development, deployment, and diffusion are the 
acquisition of enabling IT assets, the set-up of a firm's competencies, and the diffusion of a new service. Although the proposed transformation is represented as a phase-by-phase process, as shown in the case studies, the three phases may not necessarily operate sequentially. Furthermore, when acknowledging that a challenge solved via inferior solutions led to inadequate fulfillment of proposed service quality, executives will definitely go back to conduct relevant activities to resolve the said challenge. That is, there are feedback loops facilitated from the competency back to the deployment phase and to the development phase, as well as from the business performance back to the diffusion phase, to the deployment phase and to the development phase. For each phase, we can comprehensively describe its associated challenges, values and expenses as follows.

\section{The development phase}

Once an IIS initiative is approved by the top management level, the first major tasks are to formulate a business-IT integration plan and then, based upon the integration plan, to acquire (or develop) the IT assets that enable the new service. These IT assets should support front-, middle-, and back-office activities to help customers take an active role in the self-service process and to ensure consistent quality and efficiency of the new service system. There are different types of IT assets comprising a portfolio of tangible inputs, including hardware, software, infrastructure, communication technology, and other complimentary intangible elements, such as knowledge, enhanced technological and managerial skills in the use of IT, among others. During this phase, the main involved unit is the IT department; infusing IT expenses (ExpenselT) is necessary for the acquisition of enabling IT assets. The level of ExpenselT impacts the breadth (i.e., number of IT assets) and the depth (that is, levels of activities supported) of acquired IT assets and thus the quality and efficiency of new service system.

It should be noted that each approved IIS proposal has been assigned a hypothesized value (ValueH) of the new service. ValueH is later tested through pilot studies of acquired IT assets to provide the firms a better understanding of ValueP, the potential value of acquired IT assets generated under optimal organizational and environmental conditions for the new service. ValueP may not equal ValueH when the acquired IT asset does not provide the hypothesized functionality. That is, there are technical challenges related to the way in which the firm converts ExpenselT into the IT asset. Although tougher technical challenges can be addressed by infusing more ExpenselT, sometimes they cannot be effectively addressed with a constrained budget; in this case, a decision to terminate or reshape the IIS initiative should be made. Moreover, with the same level of ExpenselT, some firms may be able to acquire more IT assets than others due to superior capacity to address certain technical challenges. In sum, a thorough
business-IT integration plan should be regarded as the foremost concern in the IT asset acquisition phase and a major challenge is to acquire reliable IT assets through reasonable levels of ExpenselT.

\section{The deployment phase}

After acquiring IT assets, the firm should incorporate them into the proposed service system and create competencies via a synergy of cross-functional capabilities of different functions. That is, the firm should find an effective way of integrating acquired IT assets with other service components, such as service concepts and people and processes involved in the front-, middle-, and back-offices. Thus, there is a deployment phase that involves the building up of a firm's competencies including effective use of IT in service processes and activities, improved understanding of service markets and customers and flexible and adaptive organization in support of service development and delivery.

At the deployment phase, there are managerial and organizational challenges that undermine this competency and prevent the firm from delivering what the consumer expects; thus, managerial expenses (expenses managerial) are further required to develop a proper foundation for the new service. Expenses managerial refers to the utilization of the organization's capabilities by leveraging all of its business resources, including tangible resources (for example manpower, management teams and training) and intangible resources (for example culture, incentives and reputation). For instance, executives in the field of online logistics services are aware that radio frequency identification (RFID) technology has a high potential to provide efficient tracking of parcels, a vital component of high-quality shipping services.

However, providing such a shipping service may be difficult due to a generalized lack of trained service personnel, an absence of total management commitment, inefficient business processes, and an unsupportive corporate culture.

In this phase, the main involved units are top management and non-IT departments; it is necessary to formulate a deployment strategy to infuse Expenses managerial into the deployment project and to manage the deployment project effectively. The proper utilization of resources and effective coordination and integration of all capabilities will serve as a basis for business competency in the evolution of the new service. With service component integration and testing activity, the firm becomes more aware of the IIS project outcomes vis-à-vis customer expectations. In other words, ValueP gets transformed into ValueE, the expected value that may be gained before new service diffusion.

\section{The diffusion phase}

Via the firm's competencies, a new service diffuses to the 
public to realize its value. Hereafter, ValueR refers to the real value gained from the market. Normally, the diffusion of a new service presents additional managerial challenges; for example, the firm needs to learn about new markets and tune up the service interaction and relationship processes accordingly. Inefficient setup resources, such as legacy systems, legacy processes and absent knowledge regarding new markets, often prevent the firm from maximizing ValueR. Additionally, an inadequate organizational performance may obtain when the firm is in an unfavorable environment (for example, dealing with ambiguous laws discouraging the diffusion of new service), in an ineffective partnership in helping provide new service, in a competitive environment that makes the new service unattractive, or in a scenario of low customer acceptance. The firm may need to develop a favorable environment through some deliberate activities that, for example, help diffuse the new service with either greater benefits (for example resulting from a larger customer base) or lower costs (for example resulting from a more efficient diffusion process) in comparison with its competitors.

In this phase, therefore, expenses market, consisting of a variety of social/environmental, inter-organizational and managerial expenses, is required to improve ValueE. That is, expenses market is required to cope with: (1) Social/environmental issues, including gaining reference group acceptance and government support, (2) interorganizational issues, entailing the strategies for satisfying key stakeholder needs, seeking trustworthy partners, maintaining good governance and understanding competitor strategies and (3) managerial issues, such as designing and maintaining an efficient process for diffusing new services, building high market sensing capabilities and responding proactively to market trends.

\section{THE VALUE-EXPENSE PERCEPTION}

From the conducted case studies, we further recognized that each of the following variables, ExpenselT, expense managerial and expense market, consists of the expenses incurred to date (Expensel) and the expected expenses incurred in the future (ExpenseF). ExpenseF is attributed to some technical, managerial or market challenges that had been coped with via bad timing or inadequate expenses such that the resulting service quality is inferior and certain service continuance activities and extra expenses are required.

Moreover, it is difficult to conduct a formal analysis of each value and expense since many uncertainties exist in measuring ValueC, ValueCA and ExpenseF due to the ambiguities in required IT assets, required competencies, reference group, needs of the reference group and the social/business environment. Even so, there is a valueexpense perception at any time period of three-Ds of IIS, regardless of the presence of formal value-expense analyses. The value-expense perception evolves periodically when there is a better idea about IT assets, competencies, the reference group, needs of the reference group, and the social/business environment. For instance, executives may suspect that certain challenges (that is, full-scale change management or fullscale fulfillment of customer needs) lead to an unaffordable ExpenseF.

In the conducted case studies, we find that certain perceptions such as too much ExpenseF and a big depreciation of ValueR cause executives to be cautious, pushing them to stop the realization processes of new iTV services. In the Time Warner case, the high cost of set-top-box equipment, the low usage rates, and low revenues caused top management to be cautious, this in turn led to the termination in the diffusion of the new service. The high cost of set-top-box equipment implies a high cost of acquiring the new service, thus leading to a perception of low ValueR. Having a reasonable cost of set-top-box equipment was crucial in obtaining a critical mass of customers for a sustainable new service; however, it seems that a high ExpenseF in terms of IT asset acquisition is to be expected. On the other hand, both low usage rates and low revenues imply a low degree of addressing customer needs of the reference group, a low process quality of the way the new service was delivered, a misalignment between perception and expectations of the reference group regarding the new service and high costs of acquiring the new service. Such low usage rates and low revenues also lead to the perception of a low ValueR of the new service and a high ExpenseF when the new service is implemented in full scale.

In the TTV case, the top management faced lots of managerial and market challenges that render them hesitant to deploy and diffuse the iTV project. iTV service requires interactions not only among people, products and technologies but also within the content production food chain of the iTV service (Figure 2) that may involve intra- and inter-organizational business units. This implies that the top management needs to pay attention to the likelihood of having a high ExpenseF for iTV service system integration. Furthermore, the firm is likely to have a high ExpenseF due to significant efforts in changing the management if top management wants to push the content production food chain of iTV services into fullscale implementation.

In both iTV cases, the following market challenges also lead to a certain perception of a low ValueR and high ExpenseF regarding iTV service promoted by cable or terrestrial television companies. First, in a TV platform involving a mass media platform shared with many content providers and platform operators, the transparency of service systems makes imitation simpler and patents more difficult to obtain. These lead to concerns for top management vis-à-vis a potential scenario of a low ValueC and ValueCA. Second, because customers participate in the ITV service systems, 
innovation tends to be evolutionary rather than radical in allowing for acceptance. This leads to worries about the possibility of a lower ValueC and ValueCA when iTV service is radical. Third, iTV services cannot be realistically tested in an isolated laboratory/environment, so they run the risk of failure or poor performance upon launch. It points to a possibility of having a very high ExpenseF. Fourth, there is a complex value net (Figure 3 ) in the TV broadcasting industry. This leads to concerns over a high ExpenseF in terms of collaboration in the market. Fifth, it is difficult to identify the reference group that is willing to pay a significant amount to watch limited iTV programs offered by but a few TV broadcasters. Thus, it is difficult to form a reasonable business model for the iTV service.

\section{MANAGERIAL IMPLICATIONS}

From the present study that sought to provide a comprehensive picture of the potential challenges of three-Ds of IIS and explain how both value and expense are transformed within the processes, we can conclude that elaborate ITeS enable service-for-service exchanges and can produce innovations and challenges stated in Barrett and Davidson (2008). However, ITeS have to be elaborate in order to not only cope with recoils from the impact on all levels; societal, industry, profession, organizational, group and individual, but also to maximize both customer and firm values while keeping their expenses under control within three-Ds of IIS. The implications for organizations, their executives and employees are as follows:

1. Operation: Every organization may have an ambitious IIS project to achieve competitive advantages, albeit with limited tangible and intangible resources. It is better to take proactive action in efficiently and effectively allocating resources to cope with the challenges in acquiring proper IT assets, setting up sufficient competencies and obtaining better business environments. Normally, extra resources should be prepared.

2. Business model: To have reasonable ValueC and ValueCA, the business model of new ITeS should be based on a well-defined market segment of customers that are willing to pay and to be trained as active participants in the self-service process.

3. Focus and strategic partners: To have a cost-effective business environment for new ITeS, it is better to organize a simplified and effectively integrated information chain in which the firm's position should be simple and clear. Moreover, having significant service enhancements by the participating firms in the information chain requires binding commitments, specialized knowledge and expertise and collaborations amongst the partners. They must be committed to building the infrastructure, which includes the establishment of training programs that impart the necessary skills and the establishment of proactive marketing disciplines. Governance for ITeS partners can be established through a third-party enforcement of agreements (for example, legal contracts) or by selfenforcing agreements.

4. Organizational mindset: A commitment to radical IIS means a change in the firm's current strategies, which will influence the structure, processes, employee skills and also reward systems (Clark et al., 1997; Galperin and Bar, 2002) while leading to a creative self-destruction of the organization despite a resistance to change. Especially when a firm's current profitability is ensured and there is no external competition, an organization may be willing to consider the adoption of incremental ITeS enhancements only if current market participants also offer them.

5. Perception: There are no solid theories for measuring the value and expense of IISs since the dramatic evolution of IT/IA results in dynamics and thus uncertainties, in ValueC, ValueCA and ExpenseF. Nevertheless, the proactive management of three-Ds of IIS, and the outcome and subsequent actions of the IIS project, are driven by a value-expense perception. It is better for executives to develop a strong and reasonable perception of value and expense of IISs.

Overall, ITeS need an elaborate concept for the proactive management of the realization process based on providing a prosperous guarantee. The study also provides IT professionals with the following implications regarding IISs:

1. IISs are more complex than traditional IT-related investments due to service characteristics such as coproduction, participation, simultaneity, intangibility and perishability. These characteristics greatly increase the breadth and depth of the complexity in enabling IT assets for not only service encounters and front-office activities, but also for middle- and back- office activities.

2. The acquisition of enabling IT assets is a key issue in new service. Setting up competencies for the news services to guarantee successful diffusion requires a building up of proper complementary assets (for example, business models, collaborations and work practices in service provision).

3. While traditional IT-related investment projects focus on IT-enabling benefits (that is, efficiency and cost down for the firms) at the inter- and intra-organizational levels, IISs emphasize IT-enabling benefits (that is, convenience and efficiency for customers, efficiency and cost down for the firms) at the customer, inter-, and intra-organizational levels. Furthermore, IISs need to take into consideration IT-enabling issues (for example, customer psychology, law and regulation) at the social/environment levels more seriously. For instance, while traditional IT-related 
investment projects try to control a firm's expenses, IISs need to take into consideration not only the firm's expenses but also the customers' costs of acquiring the services.

The conducted case studies in this present study do not cover the phase of service design. A future work is planned that will have an in-depth case study focusing on the service design phase. This study does not focus on the issues surrounding appropriate levels of resources needed for IISs, how and when to collect such resources, the maximization of the value of IISs, or risk reductions in potentially incurring a skyrocketing ExpenseF under the constraints of having limited resources. All of these areas warrant further investigation.

\section{REFERENCES}

Apte UM, Goh C (2004). Applying lean manufacturing principles to information-intensive services. In. J. Serv. Tech. Manage. 5(5/6):488506.

Barrett M, Davidson E (2008). Exploring the Diversity of Service Worlds in the Service Economy, Information Technology in the Service Economy: Challenges and Possibilities for the 21st Century. In Barrett M, Davidson E, Middleton C and DeGross J(eds.), Boston: Springer. pp. 1-10.

Baumohl B, Greenwald J (1997) Time Warner's big turnoff. Time 149(19):167.

Blank C (1995). The FSN challenge: Large-scale interactive television. Computer 28L(5):9-12.

Britannica (2010). http://www.britannica.com/EBchecked/topic/765198/ Time-Warner-Inc,

Bruhn M, Georgi D (2006). Services marketing: managing the service value chain. Essex. England: Prentice Hall.

Clark CE, Cavanaugh NC, Brown CV, Sambamurthy V (1997). Building Change- readiness Capabilities in the IS Organization: Insights from the Bell Atlantic Experience. MIS Q. 21(4):425-454.

Elmer-Dewitt P, Woodbury R (1994). Ready for prime time? Time 144(26):125-126.

Farhi P, Corcoran E (Dec. 13 1994). Interactive in Orlando: Data Highway Gets a Consumer Acid Test. Washington Post. A1:22.

Fitzsimmons JA, Fitzsimmons MJ (2008). Service Management: Operations, Strategy, Information Technology. New York: McGrawHill.

Galperin H, Bar F (2002). The regulation of interactive television in the United States and the European Union. Fed. Comm. Law J. 55(1):6184.

Gronroos C (1990). Service Management and Marketing: A Customer Relationship Management Approach. Lexington, Mass: Lexington Books.
Heskett JL, Sasser WE, Schlesinger LA (1997). The service profit chain: how leading companies link profit and growth to loyalty, satisfaction, and value. New York: Free Press

Karmarkar U, Apte UM (2007). Operations Management in the Information Economy: Information Products, Processes, and Chains. J. Oper. Manage. 25(2):438-453.

Kohli R, Sherer SA (2002). Measuring Payoff of Information Technology Investments: Research Issues and Guidelines. Comm. Acad. Inform. Syst. 9:241-268.

Lovelock CH, Wirtz J (2010). Service Marketing ( $7^{\text {th }}$ edition). N.J.: Prentice Hall.

Meyer K, Fahnrich K (2009). "How to 'Engineer' IT-Enabled Services". Proc. of 1st International Symposium on Services Science ISSS'09, Logos, Berlin. pp. 137-147.

Ministry of Economic Affairs (MOEA) (2006). Information Technology enabled service (ITeS). White Paper on Taiwan Industrial Technology, Part II, Section 20. Available at http://white.tier.org.tw/2006/English/index.htm. [Accessed: 26 Feb. 2011].

Rai A, Sambamurthy V (2006). Editorial Notes-The Growth of Interest in Service Management: Opportunities for Information Systems Scholars. Inform. Syst. 17(4):327-331.

Regan WJ (1963). The Service Revolution. J. Market. 27(3):57-62.

Sasser E, Olsen RP, Wyckoff DD (1978). Management of Service Operations. Boston: Allyn and Bacon.

Schwalb EM (2004). iTV Handbook: Technologies and Standards. N.J.: Prentice Hall.

Sheth A, Verma K, Gomadam K (2006). Semantics to energize the full services spectrum. Comm. ACM. 49(7):55-61.

Soh C, Markus ML (1995). How IT Creates Business Value: A Process Theory Synthesis. In the Proceedings of 1995 International Conference on Information Systems (ICIS).

Teece D (1986). Profiting from technological innovation: Implications for integration, collaboration, licensing and public policy. Res. Policy 15(6):285-305.

Tsaih R, Chang HL, Huang CY (2005). The business concept of utilizing the interactive TV. Indus. Manage. Data Syst. 105(5):613-622.

Vargo SL, Lusch RF (2004). Evolving to a new dominant logic for marketing. J. Market. 68:1-17.

Warner Communication Annual Report (1983). Available from: http://deconstructingpopculture.com/2009/04/warnercommunications-annual-reports/. [Accessed at: 20 Jan. 2011].

Wikipedia (2011). Full Service Network. Available from: http://en.wikipedia.org/wiki/Full_Service_Network. [Accessed at: 12 Jan. 2011].

Zeithaml VA, Bitner MJ (1996). Services Marketing. New York: McGraw-Hill. 